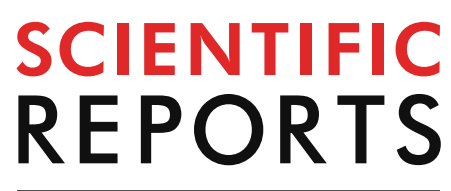

natureresearch

Check for updates

\title{
Mass spectrometry imaging as a potential technique for diagnostic of Huanglongbing disease using fast and simple sample preparation
}

\author{
João Guilherme de Moraes Pontes ${ }^{1}$, Pedro Henrique Vendramini ${ }^{2,3}$, Laura Soler Fernandes ${ }^{1}$, \\ Fabricio Henrique de Souza ${ }^{4}$, Eduardo Jorge Pilau, ${ }^{5}$ Marcos Nogueira Eberlin ${ }^{2,3}$, \\ Rodrigo Facchini Magnani ${ }^{6}$, Nelson Arno Wulff ${ }^{6}$ Taicia Pacheco Fill ${ }^{1 凶}$
}

Huanglongbing (HLB) is a disease of worldwide incidence that affects orange trees, among other commercial varieties, implicating in great losses to the citrus industry. The disease is transmitted through Diaphorina citri vector, which inoculates Candidatus Liberibacter spp. in the plant sap. HLB disease lead to blotchy mottle and fruit deformation, among other characteristic symptoms, which induce fruit drop and affect negatively the juice quality. Nowadays, the disease is controlled by eradication of sick, symptomatic plants, coupled with psyllid control. Polymerase chain reaction (PCR) is the technique most used to diagnose the disease; however, this methodology involves high cost and extensive sample preparation. Mass spectrometry imaging (MSI) technique is a fast and easily handled sample analysis that, in the case of Huanglongbing allows the detection of increased concentration of metabolites associated to the disease, including quinic acid, phenylalanine, nobiletin and sucrose. The metabolites abieta-8,11,13-trien-18-oic acid, suggested by global natural product social molecular networking (GNPS) analysis, and 4-acetyl-1-methylcyclohexene showed a higher distribution in symptomatic leaves and have been directly associated to HLB disease. Desorption electrospray ionization coupled to mass spectrometry imaging (DESI-MSI) allows the rapid and efficient detection of biomarkers in sweet oranges infected with Candidatus Liberibacter asiaticus and can be developed into a real-time, fast-diagnostic technique.

Huanglongbing (HLB) disease affects different species of citrus, such as lemons, limes, oranges and mandarins. The disease causes the development of small and defective or lopsided fruits, while leaves show blotchy mottle, resulting in development of yellow shoots. Twig dieback, stunting, and tree decline are common symptoms in the affected trees ${ }^{1,2}$. The etiological agent of HLB disease is the bacteria Candidatus Liberibacter spp. This pathogen is transmitted when inoculated in the sap of citrus plants through the vector Diaphorina citri or Trioza erytreae and by grafting ${ }^{3,4}$. The etiological agent is widespread, so the species are named in accordance to the continent where they were initially identified (Candidatus Liberibacter asiaticus (CLas), Candidatus Liberibacter americanus (CLam), Candidatus Liberibacter africanus (CLaf)), and Candidatus Liberibacter europaeus (CLeu $)^{1,5-7}$. Currently the disease is responsible for the greatest economic loss in the citrus industry ${ }^{8,9}$.

\footnotetext{
${ }^{1}$ Laboratório de Biologia Química Microbiana (LaBioQuiMi), IQ-UNICAMP, Campinas, SP, Brazil. ${ }^{2}$ ThoMSon Mass Spectrometry Laboratory, IQ-UNICAMP, Campinas, SP, Brazil. ${ }^{3}$ MackMass Laboratory, School of Engineering - PPGEMN, Mackenzie Presbyterian University, São Paulo, SP, Brazil. ${ }^{4}$ Programa de Pós-graduação Em Ciências Biológicas, Universidade Estadual de Maringá, Maringá, PR, Brazil. ${ }^{5}$ Departamento de Química, Laboratório de Biomoléculas E Espectrometria de Massas (LabioMass), Universidade Estadual de Maringá, Maringá, PR, Brazil. ${ }^{6}$ Departamento de Pesquisa \& Desenvolvimento, Fundo de Defesa da Citricultura (FUNDECITRUS), Araraquara, SP, Brazil. ${ }^{\varpi}$ email: taicia@unicamp.br
} 
The HLB disease has no cure, therefore diseased plants need to be eradicated as a control method ${ }^{10,11}$. Scouting is the way field inspector finds the symptomatic plants in the orchards. Symptoms are recognized and based on the training of the scouts. However, tree detection based on symptoms is inefficient, requiring several passes of diverse team to find all symptomatic trees ${ }^{12}$. The development of a faster and effective diagnostic tool for HLB disease is crucial to prevent the spread of the disease. Currently, the diagnosis of the disease in the symptomatic phase is mainly performed by the polymerase chain reaction (PCR) technique. However, the PCR methodology is expensive and demands a time-consuming sample preparation. Furthermore, the evaluation of HLB disease through CLas detection is negatively affected by its irregular distribution in leaves and by the low concentration of CLas cells during the initial phase of the latent period. In addition, the technique has limitations in differentiating viable and dead bacterial cells from each other, leading to an inaccurate quantification ${ }^{13-15}$. Efforts have been made in order to have a successful disease diagnostic through other analytical platforms, such as nuclear magnetic resonance (NMR) spectroscopy ${ }^{16,17}$, infrared spectroscopy (IV $)^{18}$, capillary electrophoresis with diode array detection (CE-DAD) ${ }^{19}$, differential mobility spectrometry (DMS) ${ }^{20}$, and confocal Raman microscopy ${ }^{21}$. Despite this, these techniques sometimes require a time-consuming sample preparation methodologies and generate complex data that need a time-demanding statistical data treatment (spectroscopic methods) or final adjustments for spatial resolution (microscopic methods) ${ }^{22-25}$.

Mass spectrometry imaging (MSI) enables variations of metabolites to be identified on tissue sample regions on the cellular or subcellular level. This special feature allows new possibilities in chemical biology research ${ }^{26,27}$. MSI analyses are suitable for complex surfaces because it allows the detection of hundreds to thousands of compounds, such as lipids, proteins, and low molecular weight molecules. Furthermore, MSI indicates the spatial distribution of compounds in the sample surface ${ }^{28-31}$.

MSI was applied to analyze leaves from Citrus sinensis grafted on Citrus limonia infected by Xylella fastidi$o s a$. The technique was used with success to monitor the presence of hesperidin and rutin in the infection site s. $^{32}$. MSI was also applied to monitor other plant diseases ${ }^{33-35}$; however, this technique has never been applied to understand the infection process of CLas in citrus. Herein, we applied for the first time MSI analyses combined with HPLC-MS/MS to investigate healthy and diseased leaves (both asymptomatic and symptomatic stages) in the search for HLB biomarkers. We believe that these results may further help understanding the pathogenicity of CLas.

\section{Materials and methods}

Sample collection. Valencia sweet orange (Citrus sinensis (L.) Osbeck) grafted in Rangpur lime (Citrus limonia Osb.) rootstocks infected by grafting with budsticks carrying CLas, were kept in the greenhouse under standard phytosanitary and nutritional conditions, protected from the outside by screen, avoiding the entrance of insect vectors. The greenhouse contained a pad-fan to avoid excessive heating, but temperature fluctuations still occurred in correspondence to the environmental conditions. Healthy and CLas-infected plants were pruned 13 months before sampling and allowed to grow. PCR-positive trees showed typical blotchy mottle in older leaves, while younger leaves from PCR-positive trees were mostly asymptomatic. In the sampling analyses carried out before pruning, it was found that CLas was detected in leaves of each tree and none in the healthy tree. DNA was extracted from midribs of the leaves ${ }^{36}$ and CLas was detected using hydrolyzing probes marked with 5-FAM and quenched with BHQ (Macrogen, Seoul, Korea) according to Li et al. ${ }^{37}$, considering as positive samples with Ct below 35.0 and negative those with Ct values above 35.

Leaves at stage $\mathrm{V}^{38}$ were collected directly in the greenhouse from five healthy plants (negative in qPCR analysis), three asymptomatic but CLas infected-plants confirmed by qPCR, and five symptomatic plants (CLas infected confirmed by qPCR). The leaf samples were placed inside individual plastic bags and stored at $4{ }^{\circ} \mathrm{C}$ until analyses. Healthy and CLas-infected plants were subjected to the same cultural practices, with the same scion/ rootstock combination, and from the same age, handling, storage and nutritional treatments ${ }^{39,40}$ in order to avoid metabolic changes in the leaves that were not related to the infection process. The diseased status of the plants was the only contrasting characteristic between healthy and CLas-infected. Symptomatic samples were selected based on the presence of blotchy mottle, the most conspicuous symptom displayed by HLB-infected plants. Asymptomatic leaves were from the CLas-infected trees, but without any typical HLB symptom.

DESI-MSI analyses. The leaf preparation for DESI-MSI analyses was based on scientific literature that reviews methodologies for sample preparation of plants such as Arabidopsis thaliana and Lotus japonicus ${ }^{26,27}$. Firstly, the abaxial side of the leaves were lightly scraped with a small scalpel, cut in square format, and glued with tape on the glass plate (Figures S1 to S8 in Supplementary Information). Each horizontal row was immediately analyzed to avoid dryness of the sample, which would impair the analysis. The plates were prepared in accordance to Fig. 1, and the analyses were performed four times with different samples from the same treatment.

The analyses were performed in a Thermo Scientific Q-Exactive MSI using desorption electrospray ionization (DESI) source (Omni Spray 2D-3201 model-Prosolia, Indianapolis, USA). The values range of mass-to-charge $(\mathrm{m} / \mathrm{z})$ scanned was between 100 and 1,000 , both in positive and negative modes. Other parameters adjusted were spray voltage of $1.5 \mathrm{kV}$, capillary temperature at $250{ }^{\circ} \mathrm{C}$, S-lens of 100.00 , pixel scan of $200 \mu \mathrm{m}$, resolution of $17,500(\mathrm{~m} / \Delta m \text { at } m / z 200)^{41}$, flow rate of $5 \mu \mathrm{L} \mathrm{min}^{-1}$ and a scanning rate of $740 \mu \mathrm{m} \mathrm{s}^{-1}$. In the DESI-MSI analyses conducted, a sample size of $1.5 \mathrm{~cm} \times 2.0 \mathrm{~cm}$ (length $\times$ width) for each leaf region was used. Images were processed with the use of BioMAP 3.8.0.4 (Novartis, Basel, Switzerland) software. For each $\mathrm{m} / z$ value obtained, an image was generated; all images were processed with a bin size of 4.0 and standardized with the same intensity level (5.0 in the intensity level bar). The exact mass was obtained using Thermo Xcalibur Roadmap software 3.0.63 (Waltham, MA, USA). 


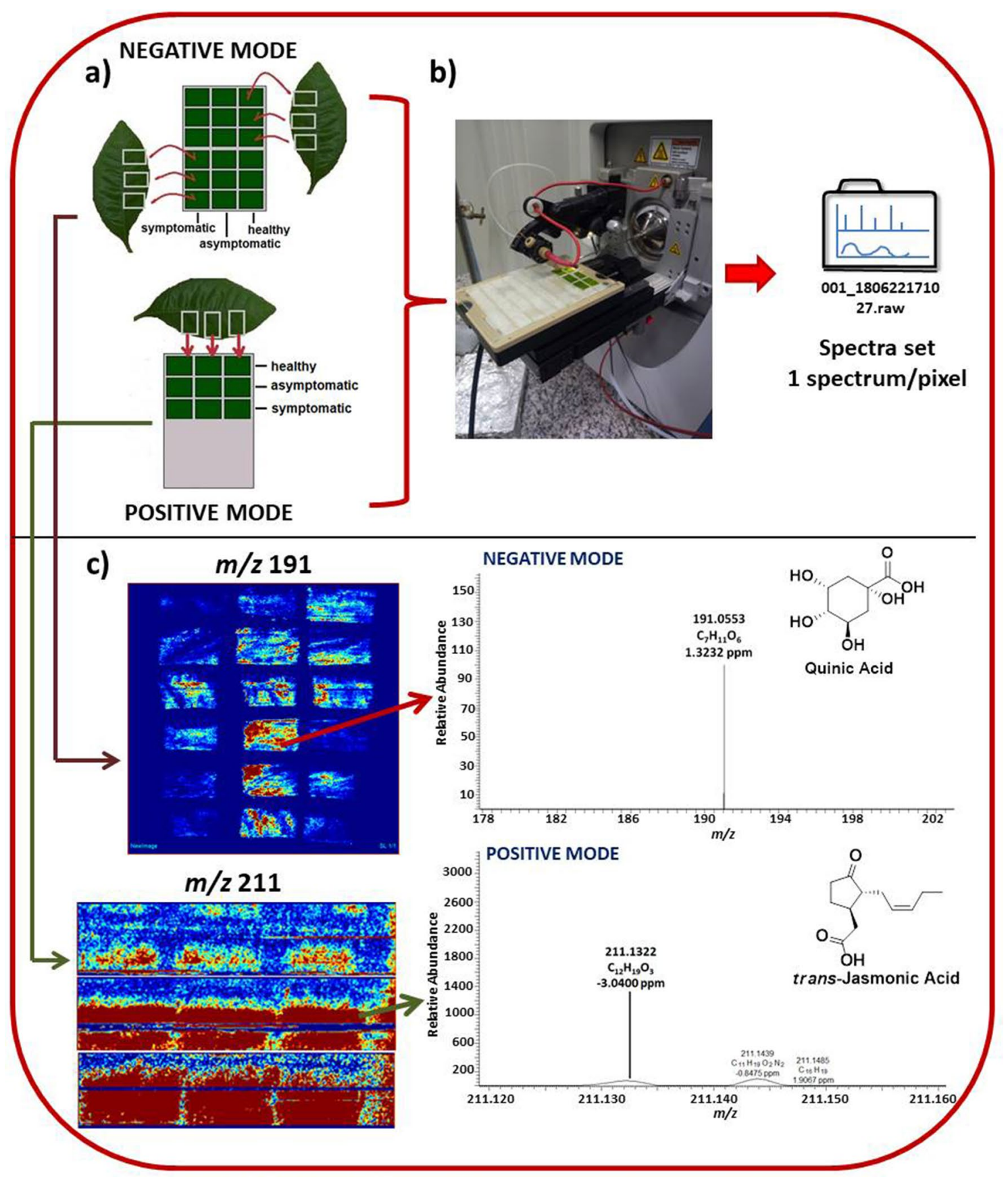

Figure 1. Image acquisition for mass spectrometry imaging of abaxial side of leaves. (a) Preparation of samples for leaf metabolite analysis on the plate in negative and positive mode; (b) scanning of leaf tissue surface and acquisition of the spectrum set; (c) DESI-MSI of the $\mathrm{m} / z 191$ that corresponds with quinic acid and $\mathrm{m} / \mathrm{z} 211$ that corresponds to jasmonic acid in negative and positive mode, respectively. The exact masses were found in Xcalibur software.

Sample preparation for LC-MS/MS analyses. The same groups of leaf samples used in previous analyses were macerated with liquid nitrogen. Three leaves from the symptomatic, asymptomatic, and healthy groups were macerated together. It was weighed $0.2 \mathrm{~g}$ of the leaves, which was extracted with $2 \mathrm{~mL}$ of different organic solvents. The solvents were tested to optimize metabolite extraction; ethyl acetate, dichloromethane, and methanol were evaluated. The samples were vortexed and were dried under a nitrogen flow in order to remove the extraction solvent. Then, the samples were dissolved in $1 \mathrm{~mL}$ of HPLC-grade methanol and filtered using a Millipore PTFE hydrophobic membrane filter with a $0.22 \mu \mathrm{L}$ pore diameter.

The HPLC-MS analyses were performed three times in order to evaluate reproducibility. The first analysis was done in negative mode with 18 leaves of each group (asympotamic, symptomatic and healthy groups), been six samples per group, totalizing 54 leaves and 18 spectra; the second analysis was done in negative mode with nine leaves of each group, been three samples per group, totalizing 27 leaves and nine spectra. The third analysis 
was done in positive mode and in duplicate with nine leaves of each group ( 3 samples/group), totalizing 27 leaves and 18 spectra (Figure S9 in Supplementary Information).

LC-MS/MS analyses. The analyses were performed using ultra performance liquid chromatography (Shimadzu, Nexera X2, Japan) coupled to mass spectrometry (Impact II, Bruker Daltonics Corporation, Germany). It was used an Acquity UPLC CSH C18 column with $2.1 \times 100 \mathrm{~mm}$ (Waters, Ireland), $2.1 \mu \mathrm{m}$ of particle size and flow rate $0.200 \mathrm{~mL} \mathrm{~min}^{-1}$. The mobile phase was composed of $1 \%$ formic acid in water $(v / v)$ and $0.1 \%$ methanol in formic acid $(v / v)$ at $40^{\circ} \mathrm{C}$.

The analyses were performed using a quadrupole time-of-flight mass analyzer (QTOF) with a collision energy ramp of $10-45 \mathrm{eV}$. The mass range $(\mathrm{m} / \mathrm{z})$ scanned was between 50 and 1,400 with an acquisition rate of $5 \mathrm{~Hz}$ for MS and $7 \mathrm{~Hz}$ for MS/MS in positive mode $[\mathrm{M}+\mathrm{H}]^{+}$with end plate offset potential $-500 \mathrm{~V}$. The four most intense ions were selected for automatic fragmentation (AutoMS/MS). Spectra were processed in DataAnalysis 4.2 software.

Metabolites identification. The molecular formulas obtained in addition to the MS/MS fragmentation pattern were submitted to different databases for identification. The results obtained were compared with human metabolome database $(\mathrm{HMDB})^{42}$, global natural product social molecular networking (GNPS) ${ }^{43}$, dictionary of natural products $(\mathrm{DNP})^{44}$, and literature ${ }^{16,45-49}$. The GNPS analyses for dereplication (or library search) were performed with precursor ion mass tolerance of 0.2 and fragment ion mass tolerance of 0.1 , which are selected parameters in the GNPS related to mass tolerance and how much the fragment ion can be shifted from expected $\mathrm{m} / \mathrm{z}$ values, respectively. For molecular networking, it was used precursor ion mass tolerance of 2.0, fragment ion mass tolerance of 0.5 , and a minimum cosine score (min. pairs cos.) of 0.7 . In dereplication the search for similarity of mass spectra is done based on databases available in the GNPS, while molecular networking enables discover new molecules and chemical species from databases and data submitted by users of the GNPS.

\section{Results and discussion}

Sample preparation and image acquisition. HLB disease was responsible for the eradication of approximately fifty million orange trees (100,000 ha) from 2004 to 2018 in São Paulo state, Brazil ${ }^{50}$ and $72.2 \%$ reduction of orange production in United States between 2007 and 2018, implicating in huge losses for worldwide citriculture ${ }^{51}$.

Currently, HLB disease detection methods are time consuming and involve multiple stages of sample preparation. Efforts have been done to have an early diagnostic of the disease with no sucess ${ }^{16-21}$. Due to these reasons, our group has been focusing attention on HLB disease and a new potential diagnostic method. Therefore, in order to monitor the metabolic profile of healthy leaves from Citrus sinensis compared to the diseased metabolic profile in asymptomatic and symptomatic stages of CLas infection, we applied for the first time mass spectrometry imaging technique. These studies would potentially contribute to helping understand the metabolites related to plant defense process, stress response, or those potentially produced as virulence factor by the pathogen.

Sample preparation for MSI analysis is relatively simple compared to other techniques such as those used within molecular biology. Healthy, PCR-negative sweet orange nursery trees were used as controls, with Ct values above the threshold limit and most often with undetectable fluorescence in qPCR analysis ${ }^{37}$ (data not shown), while CLas-infected samples had average Ct values of 20.9-23.9 for the asymptomatic leaves and Ct 19.1-21.6 for symptomatic leaves. It is possible to extract data using a leaf tissue surface with the size ranging to millimeter to centimeters and a scan time/pixel about $0.96-1.6 \mathrm{~s}^{52-54}$, which makes MSI a potential diagnostic tool to be implemented for a real-time detection of HLB in the field. Different leaf regions were fixed on the surface of a plate (Fig. 1) in order to cover different areas of the sample surface and detect a larger number of metabolites that may be distributed throughout the leaf. Figure 1 indicates the main steps for DESI-MSI image acquisition.

Another sample preparation strategy used in our studies for imaging was the imprinting procedure ${ }^{31}$; however, we chose to perform the direct analysis in the leaves tissue due to the similar results obtained in both strategies. The direct analysis led to almost zero sample preparation and faster diagnostics.

Metabolites identification. Biomap software was used for image processing. All the images acquired by MSI were standardized to have the same coloring in a blue-red scale. The metabolites accumulated in the disease are represented by the red color. Therefore, the most intense peak (100\%) is indicated by red coloration, while other colors are proportional to its relative intensity ${ }^{55}$.

The metabolites accumulated in the affected plants that were identified (putatively annotated compoundsTable 1) showed an exact mass compatible with an elemental composition with low value of delta (below $4.5 \mathrm{ppm}$ ) for all the results.

The metabolites shown in Table 1 were previously reported in citrus by using chromatography and mass spectrometry techniques: dehydroabietic acid, ABA, 4-acetyl-1-methylcyclohexene (4-AMCH) ${ }^{56-58}$, asparagine, pipecolic acid, quinic acid $^{45}$, guaiacol ${ }^{67}$, isoleucine, $t$-JA, phenylalanine, tryptophan, valine ${ }^{46}$, feruloylputrescine, hydroxycinnamates (HCAs) ${ }^{47}$, nobiletin and tangeretin ${ }^{48}$. Other metabolites in Table 1 were identified previously by NMR spectroscopy: synephrine ${ }^{49}, \beta$-glucose, sucrose, tryptophan, tyrosine and valine ${ }^{16}$.

Based on the DESI-MSI analyses conducted for this study, it was observed an increase in the accumulation of nobiletin (Fig. 2), tangeretin, feruloylputrescine, synephrine, and asparagine after the increase of disease symptoms in leaves. These results corroborate with quantification data obtained for HPLC for the feruloylputrescine ${ }^{47}$, nobiletin, and tangeretin ${ }^{48}$ and NMR data for synephrine ${ }^{49,60}$. Furthermore, nobiletin has previously been associated as a biomarker of HLB disease in the fruit juice ${ }^{68}$. 


\begin{tabular}{|c|c|c|c|c|c|c|c|}
\hline Entry & Metabolites & $\begin{array}{l}\text { Experimental } m / z \\
\text { (Xcalibur) }\end{array}$ & Database $m / z$ (HMDB) & Error (ppm) & Mode (DESI-MSI) & $\begin{array}{l}\text { References associated } \\
\text { with HLB disease or } \\
\text { plant defense }\end{array}$ & $\begin{array}{l}\text { Figures in } \\
\text { Supplementary } \\
\text { Information }\end{array}$ \\
\hline 1 & $\begin{array}{l}\text { Abieta-8,11,13-trien-18- } \\
\text { oic acid (Dehydroabietic } \\
\text { acid) }\end{array}$ & 301.2162 & 301.2162 & 0.24 & Positive & 56 & S10-S12 \\
\hline 2 & Abscisic acid (ABA) & 265.1434 & 265.1440 & -2.77 & Positive & 57 & S13-S16 \\
\hline 3 & $\begin{array}{l}\text { 4-Acetyl-1-methylcy- } \\
\text { clohexene (4-AMCH) }\end{array}$ & 139.1117 & 139.1123 & -0.08 & Positive & 58,59 & S17-S20 \\
\hline 4 & Asparagine & 133.0608 & 133.0613 & 3.17 & Positive & 51,60 & S21-S24 \\
\hline 5 & Feruloylputrescine & 265.1547 & 265.1552 & -1.92 & Positive & 47 & S25-S28 \\
\hline 6 & $\beta$-Glucose & 179.0550 & 179.0556 & 1.59 & Negative & $16,45,61$ & S29-S31 \\
\hline 7 & Guaiacol & 125.0597 & 125.0603 & 1.47 & Positive & 62 & S32-S35 \\
\hline 8 & $\begin{array}{l}p \text {-Hydroxycinnamic acid } \\
(p \text {-coumaric acid })\end{array}$ & 165.0546 & 165.0552 & 2.24 & Positive & $47,51,63$ & S36-S39 \\
\hline 9 & Isoleucine & 132.1019 & 132.1025 & 1.10 & Positive & 45,46 & S40-S43 \\
\hline 10 & $\begin{array}{l}\text { trans-Jasmonic acid } \\
(t \text {-JA })\end{array}$ & 211.1329 & 211.1334 & -3.04 & Positive & 46,57 & S44-S47 \\
\hline 11 & Nobiletin & 403.1387 & 403.1393 & -1.05 & Positive & 48,51 & S48-S50 \\
\hline 12 & Phenylalanine & 166.0863 & 166.09 & 0.57 & Positive & $45,46,49,51$ & S51-S53 \\
\hline 13 & Pipecolic acid & 130.0863 & 130.0868 & 0.81 & Positive & 45,46 & S54-S57 \\
\hline 14 & Quinic acid & 191.0553 & 191.0556 & 1.34 & Negative & 64 & S58-S60 \\
\hline 15 & Sucrose & 343.1235 & 343.1235 & 2.89 & Positive & 16,61 & S61-S63 \\
\hline 16 & Synephrine & 168.1019 & 168.1025 & -0.15 & Positive & 49 & S64-S66 \\
\hline 17 & Tangeretin & 373.1282 & 373.1287 & -0.88 & Positive & 48,51 & S67-S69 \\
\hline 18 & $\begin{array}{l}\text { Tetramethoxyflavone } \\
\text { (TMF) }\end{array}$ & 343.1176 & 343.1182 & -1.12 & Positive & 65 & S70-S73 \\
\hline 19 & Tryptophan & 205.0972 & 205.0977 & -1.34 & Positive & 16,46 & S74-S77 \\
\hline 20 & Tyrosine & 182.0812 & 182.0812 & -0.44 & Positive & 16,66 & S78-S81 \\
\hline 21 & Valine & 118.0863 & 118.0868 & 1.40 & Positive & 16,46 & S82-S85 \\
\hline
\end{tabular}

Table 1. Metabolites putatively identified using DESI-MSI ( full scan) predominantly produced in diseased samples of sweet orange leaves.

Other metabolites such as abscisic acid (ABA), asparagine, glucose, $p$-hydroxycinnamic acid, isoleucine, transjasmonic acid $(t-\mathrm{JA})$, phenylalanine, quinic acid, sucrose, tryptophan, and valine had an increase in concentration in the diseased leaves (images in Supplementary Information-Figures S16, S22, S31, S39, S43, S47, S53, S60, S63, S77, and S85, respectively), which corroborate with quantification data obtained for GC-MS (tryptophan, $t$-JA, phenylalanine, isoleucine, valine in CLas-infected ${ }^{46,69}$, GC-MS-SIM (ABA, $t$-JA and tryptophan in CLasinfected) ${ }^{57}, \mathrm{UV}-\mathrm{Vis}$ (hydroxycinnamates) ${ }^{63}$, starch assay (sucrose and glucose) ${ }^{61}$ and NMR data (asparagine and quinic acid) ${ }^{60}$. Figure 2 shows the image acquired for DESI-MSI for four metabolites (nobiletin, phenylalanine, sucrose and quinic acid) that are clearly differentiated in the diseased leaves and that were previously associated as biomarkers for HLB disease ${ }^{16,61,64,68}$, indicating that MSI technique would be a potential great tool for real-time diagnostics of HLB. The images and $\mathrm{m} / z$ obtained for each of the metabolites mentioned in Table 1 are available within the Supplementary Information (Pages S8-S55).

Flavonoids, flavones and compounds from orange. Nobiletin, tangeretin, tetramethoxyflavone, and feruloylputrescine were reported in leaf samples from Citrus sinensis (L.) Osbeck ${ }^{65,68,70}$ while synephrine and 4-acetyl-1-methylcyclohexene in orange juice ${ }^{58,60}$.

Hijaz, et al. investigated the production of tangeretin and nobiletin in HLB disease. The authors confirmed the accumulation of both metabolites in leaf samples in weeks 27 and 29, respectively ${ }^{47}$. Furthermore, Massenti et al. quantified tangeretin and nobiletin for HPLC presents in peels of Valencia orange fruits and they observed the increase of these metabolites in diseased fruits, which corroborates with our data of DESI-MSI for the leaf samples (Figures S50 and S69 in Supplementary Information) ${ }^{48}$.

$4^{\prime}, 5,6,7$-Tetramethoxyflavone (or tetramethyl-O-scutellarein) also accumulated in the infected leaves based on DESI-MSI analyses (Figure S73). This metabolite is a polymethoxyflavone (PMF), as well as, nobiletin $\left(5,6,7,8,3,4^{\prime}\right.$-hexamethoxyflavone) and tangeretin $\left(5,6,7,8,4^{\prime} \text {-pentamethoxyflavone }\right)^{65}$. The change in the concentration of PMF is based on fruit maturity and such compounds have been associated to plant defense process against pathogens ${ }^{65}$. Hijaz et al. observed an increased concentration of 4',5,6,7-tetramethoxyflavone on week 29 which is in accordance to our DESI-MSI data (Figure S73 in Supplementary Information) $)^{47}$.

Feruloylputrescine is a secondary metabolite from orange that is a conjugate of putrescine and ferulic acid ${ }^{51}$. The increase of feruloylputrescine production levels in CLas infection was quantified by HPLC-MS for Baldwin et al. in orange juice, which corroborate with our MSI data (Figure S28 in Supplementary Information) ${ }^{71}$.

Guaiacol production is a response to CLas infection based on the DESI-MSI analyses (Figure S35 in Supplementary Information), since it was observed in higher concentration in infected leaves. To the best of our 
a)
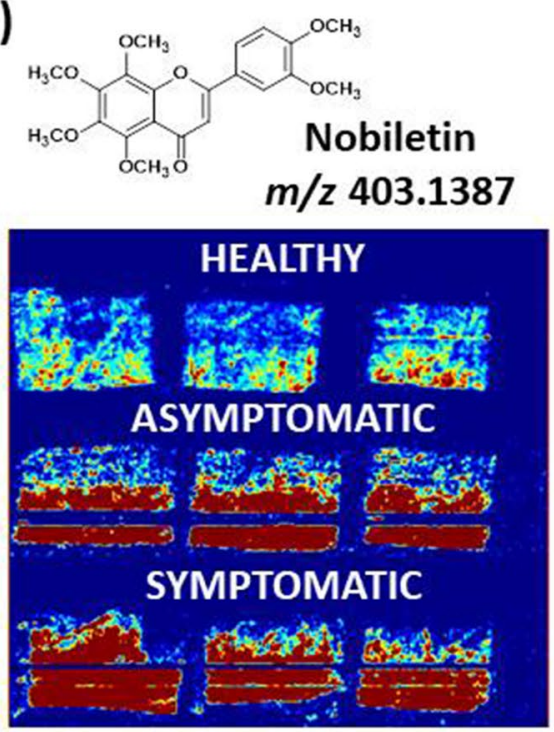

HO.

c)
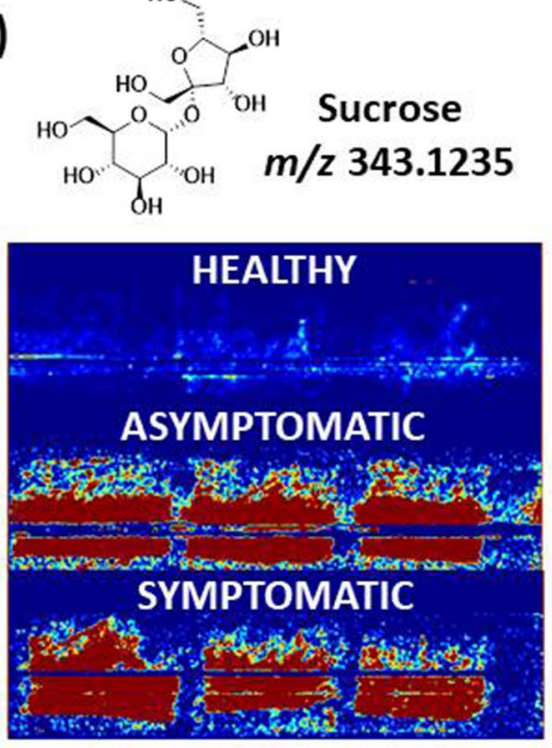

b)
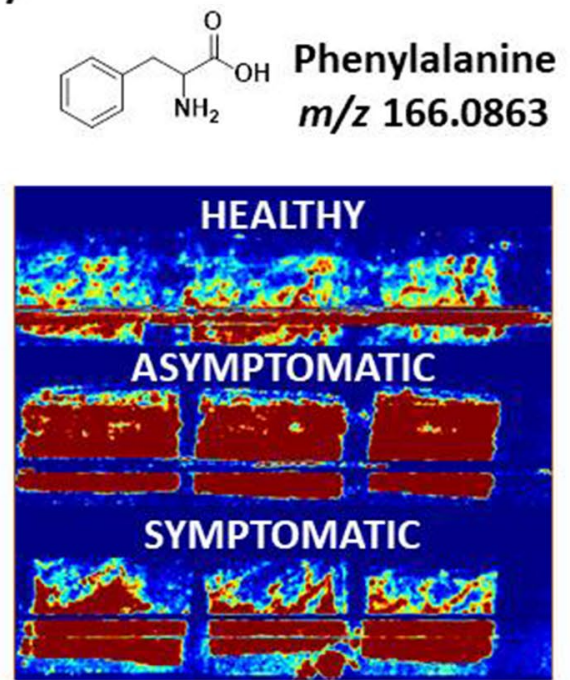

d)
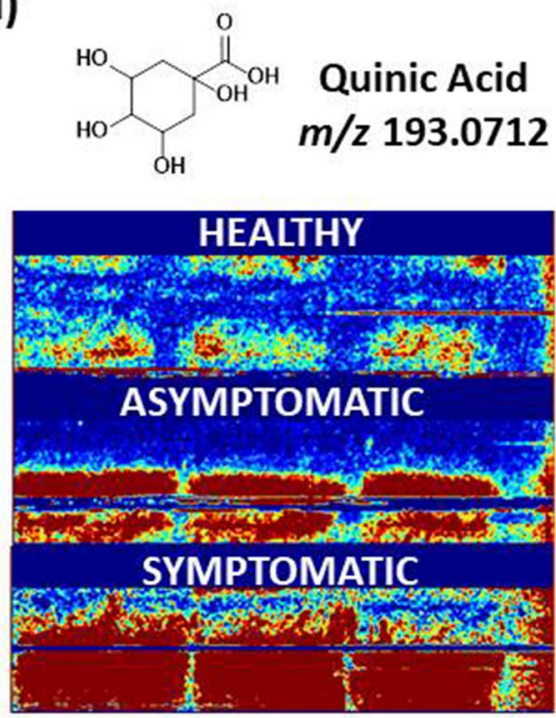

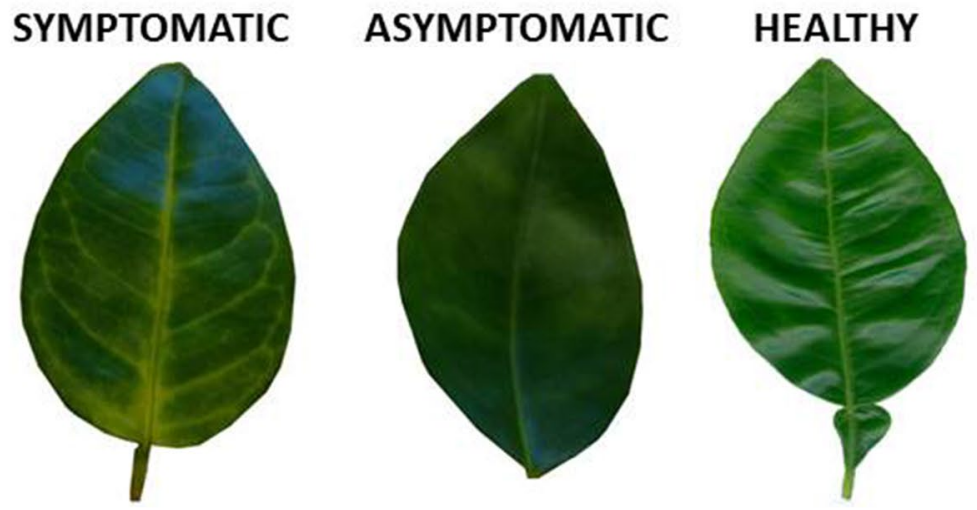

Figure 2. Biomarkers of HLB disease identified for DESI-MSI analyses in positive mode. In the images acquired, the red color indicates the ion distribution on the abaxial leaf surface in asymptomatic, symptomatic and healthy samples. The images acquired were at (a) $\mathrm{m} / z$ 403; (b) $\mathrm{m} / z 166$, (c) $\mathrm{m} / z$ 343, and (d) $\mathrm{m} / z$ 193, which the exact masses found in Xcalibur software $(\mathrm{m} / z$ 403.1387, $\mathrm{m} / \mathrm{z} 166.0863, \mathrm{~m} / \mathrm{z} 343.1235$, and $\mathrm{m} / \mathrm{z}$ 193.0712) correspond to nobiletin, phenylalanine, sucrose, and quinic acid, respectively. 
knowledge, guaiacol had never been reported as a biomarker to HLB disease. However, an increasing level of catechol O-methyltransferases (enzyme that synthesizes guaiacol) occur in infected plants ${ }^{62}$, which may result in an increase in guaiacol concentration.

Another metabolite detected by the DESI-MSI was synephrine (Figure S66 in Supplementary Information). Synephrine is an alkaloid of occurrence in Citrus of Rutaceae family and is known for its natural antihistaminic effect and ability to induce lipolysis ${ }^{72-74}$. Although the increase of synephrine levels in fruits infected by CLas is well reported in literature ${ }^{49,60}$, its role in the infection process remains unclear.

4-Acetyl-1-methylcyclohexene, a volatile oxidation product of limonene, also accumulated in the plants infected with CLas as indicated in our DESI-MS analyses. This compound is present in citric peel oil, orange juice, and other food sources ${ }^{58,75-76}$. Tao et al. verified that 4-AMCH is part of the essential oil of Ponkan (Citrus reticulata Blanco) composition, which showed antibacterial and antifungal activities against Escherichia coli and Bacillus subtilis ${ }^{77}$. So far, $4-\mathrm{AMCH}$ has not been associated to Candidatus Liberibacter spp. infection and HLB disease.

Amino acids. Asparagine, isoleucine, phenylalanine, tryptophan, tyrosine, and valine were the amino acids detected by DESI-MSI analyses, showing a higher concentration in diseased leaves (Figures S22, S43, S53, S77, S81, and S85 in Supplementary Information, respectively). Isoleucine was detected by LC-MS/MS analysis in leaf samples (Figure S41 in Supplementary Information), and this accumulation was also reported in CLas infected leaves ${ }^{46}$. The increase of valine production in CLas infected leaves and fruit was also reported ${ }^{69}$. The role of the amino acids isoleucine, valine, and tyrosine in the CLas infection process is not yet fully understood; however, it is known that CLas is incapable to produce such amino acids ${ }^{78}$. The same amino acids were detected in the flush shoots of Rutaceae host plants, flush shoots of D. citri nymphs ${ }^{66}$, and citrus leaves during defense responses to stress ${ }^{45}$.

DESI-MSI also indicated the increase of phenylalanine, tryptophan, and asparagine. Phenylalanine and tryptophan are precursors of the phytohormone salicylic acid (SA) and the auxin indole-3-acetic acid (IAA), respectively. SA and IAA are signaling compounds and may have a role in CLas as well as D. citri infection process in sweet oranges ${ }^{57}$. The increase of asparagine levels observed by DESI-MSI in Valencia oranges during the plant-pathogen interactions corroborates with literature ${ }^{59,68}$ and was reported ${ }^{66}$ as an abundant free amino acid (FAA) in permissive hosts that may be modulated by CLas.

Although DESI-MSI is able to differentiate healthy and diseased profiles through images of isoleucine, tryptophan, and phenylalanine, amino acids are not specific biomarkers for HLB disease. The variations in the levels of amino acids, as well as organic acids, are a common response during the disease process ${ }^{45-46}$.

Phytohormones. Phytohormones associated with the defense response of CLas infection and D. citri-infestation, such as ABA and $t$-JA, are also detected at higher concentrations by DESI-MSI (Figures S16 and S47, respectively in Supplementary Information). Absisic acid is a phytohormone associated with many plant physiological functions, including, plant defense response to abiotic stress and disease tolerance in different types of plant-pathogen interactions ${ }^{57,79}$.

Jasmonic acid is a phytohormone that activates the expression of plant defense against herbivores as the Diaphorina citri $^{46,80}$. Nehela et al. detected the phytohormones (auxins, ABA, SA and $t$-JA) of Valencia sweet orange leaves by GC-MS-SIM in different stages-healthy, CLas infected, D. citri infested, and double attacked. In this study they verified that ABA and SA are related to plant defense to CLas, while that $t$-JA is associated with the D. citri infestation ${ }^{57}$.

Organic acids. Organic acids are commonly associated with plant stress ${ }^{81,82}$. This alteration in the metabolic profile of organic acids was also reported in plants infected by $C \operatorname{Las}^{47,64}$. Some organic acids, such as quinic acid and $p$-hydroxycinnamic acid, were previously detected and quantified in symptomatic leaves ${ }^{47,64}$, which corroborate with our MSI results (Figures S39 and S60 in Supplementary Information).

Quinic acid was identified in different varieties of healthy citrus ${ }^{83}$ and has been associated as a citrus leaf metabolite during defense responses ${ }^{64}$. In our analysis, the peak at $m / z 191.0550$ refers to quinic acid and the MS/ MS fragmentation pattern indicates an ion at $m / z 127.0399$ corresponding to the loss of CO and two molecules of $\mathrm{H}_{2} \mathrm{O}^{84}$. A higher concentration of quinic acid was detected by DESI-MSI (Fig. 2) in asymptomatic profile followed by symptomatic profile. The increase in quinic acid level in diseased leaves was also reported by Jones et al. ${ }^{64}$, Chin et al. ${ }^{60}$ and Yao et al. ${ }^{69}$, corroborating with our DESI-MSI data. The increase in quinic acid production in the asymptomatic plants makes it a good biomarker to HLB disease as previously suggested by Jones et al. ${ }^{64}$.

Quinic acid is a primary organic acid present in leaves of sweet orange trees and can be converted to shikimic acid. Quinic acid and shikimic acid are precursors of other compounds, such as lignin, phenylalanine, and tyrosine $e^{64,85}$. Quinic acid has been detected in semi-tolerant variety of fruits to CLas ${ }^{85}$ and against other citrus pathogens as Xylella fastidiosa ${ }^{86}$; however, its role in the defense mechanism against the CLas is still not completely clear.

In our studies, $p$-hydroxycinnamic acid was also detected by DESI-MSI technique; the correspondent images obtained indicated an increase in the production of this metabolite in both asymptomatic and symptomatic profiles. The increase in hydroxycinnamates levels in oranges' leaves during CLas infection was reported in literature $e^{47,63}$ and is associated to defense responses. The metabolite $p$-hydroxycinnamic acid is a precursor in the biosynthesis of phenylpropanoid derivatives that have antimicrobial activities and are related to plant defense $\mathrm{e}^{47,87}$.

Abieta-8,11,13-trien-18-oic acid (Dehydroabietic Acid-DHA) was identified in GNPS analyses (gold classification) and was previously isolated by Vargas et al. from nonvolatile residue of citric essential oil. It is a metabolite found in resin acids of colophonium and can be present also in natural fragrance and when oxidized 


\section{CLas-Infected leaf}

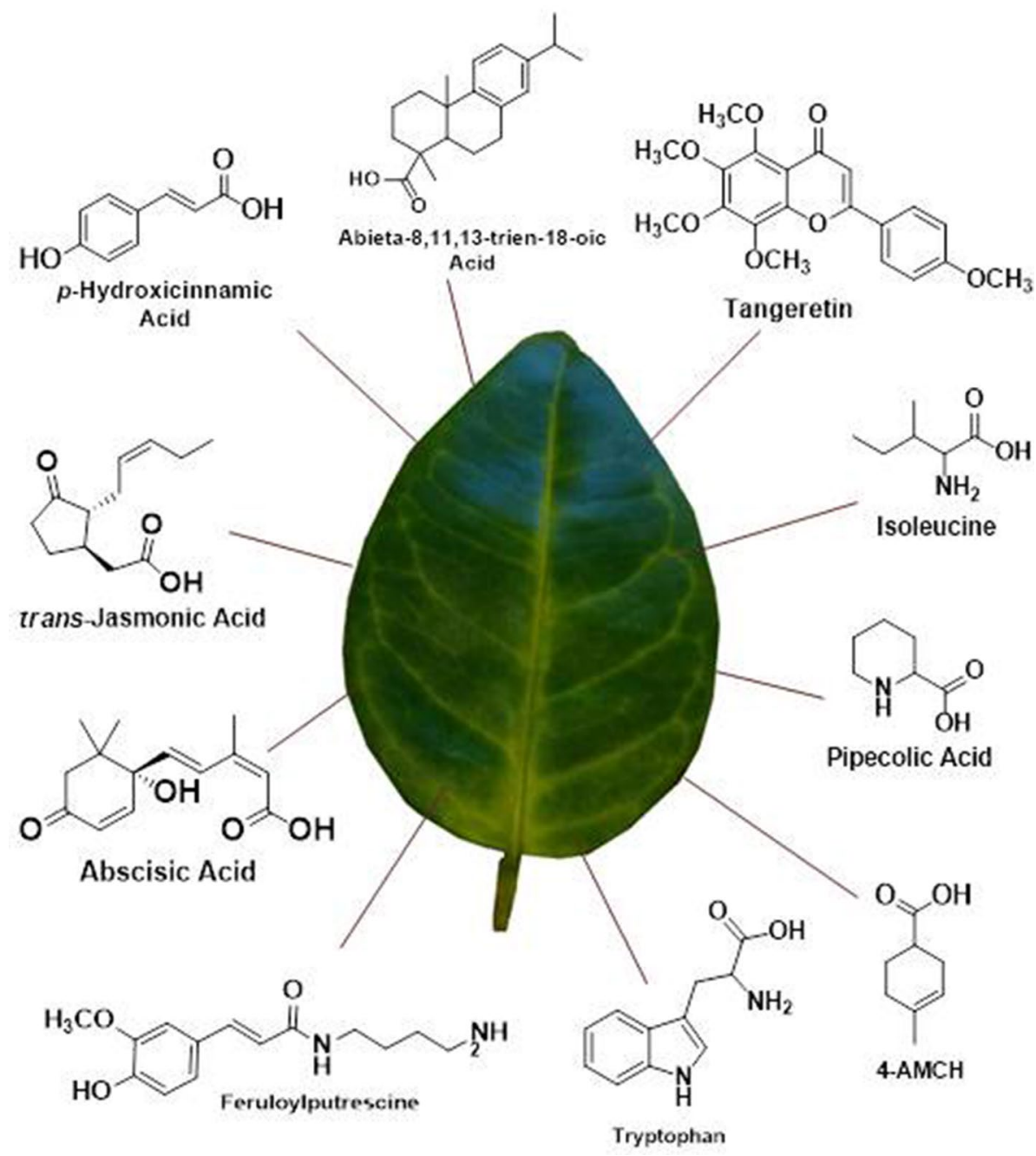

Figure 3. Potential biomarkers of HLB disease. The metabolites Abieta-8,11,13-trien-18-oic acid, abscisic acid, 4-acetyl-1-methylcyclohexene (4-AMCH), feruloylputrescine, $p$-hydroxycinnamic acid, isoleucine, transjasmonic acid, pipecolic acid, tangeretin, and tryptophan, which were detected by DESI-MSI had an increase of concentration in asymptomatic and symptomatic CLas-infected leaves. The increase of concentration is based on images acquired by DESI-MSI available in Supplementary Information (Figures S12, S16, S20, S28, S39, S43, S47, S57, S69, and S77, respectively).

can act as allergen ${ }^{88}$. This metabolite is part of pine (Pinus nigra) resin's constitution and is reported to be produced by the plant against pathogens ${ }^{89,90}$. DHA was also identified in Chamaecyparis pisifera ${ }^{91}$ and Tripterygium wilfordii ${ }^{92}$ and has been studied against various bacteria species due its antimicrobial activity ${ }^{56,93,94}$. However, this metabolite has never been associated with CLas infection, therefore more studies need to be performed to understand its role in HLB disease.

Another organic acid detected by DESI-MSI in both asymptomatic and symptomatic leaves was pipecolic acid $^{46,95}$, that is biosynthesized from lysine and acts as an immune signal regulating the plant defense responses. Pipecolic acid has been suggested in literature ${ }^{46,96}$ to participate in the defense against CLas, inducing the SAmediated pathway.

Sugars. Sugar, such as sucrose and glucose, levels increasing in leaves infected by CLas can be observed in the images of DESI-MSI analyses (Figures S31 and S63 in Suplemmentary Information) and the higher levels are in accordance with previous studies ${ }^{61,97}$. During the infection process, sucrose and glucose act as signaling compounds, inducing the feedback inhibition of photosynthesis ${ }^{61,98}$.

Figure 3 summarizes the major metabolites identified for DESI-MSI analyses involved in the HLB disease. 
Mass spectrometry imaging proved to be a suitable technique to identify a range of metabolites regulated during the CLas-sweet orange interaction, identify compounds produced in higher levels in both the asymptomatic and symptomatic stages, and indicate potential biomarker candidates with potential to develop an early diagnostic of HLB disease. Furthermore, MSI analysis showed to be a fast technique with little sample preparation and manipulation, fulfilling requirements compatible with a diagnostic process. There are examples of the accumulation of compounds in the healthy leaves as shown in Figure S86 in Supplementary Information; however, here we discussed the metabolites that had an increase in the diseased leaves and could be potential biomarkers.

Quinic acid, phenylalanine, nobiletin, and sucrose are metabolites that were previously reported in literature as biomarkers of HLB disease in orange fruits ${ }^{16,61,64,68}$. In addition to metabolites associated to plant defense or stress such as quinic acid, $t$-JA and tryptophan ${ }^{45,57}$ were also detected by DESI-MSI in asymptomatic leaves of Citrus sinensis with significant difference of healthy profile (Figs. 2 and S47 and S77 in Supplementary Information). We believe that these metabolites could be a potential parameter for infection recognition still in the asymptomatic stage; however, further validation to use the DESI-MSI as a diagnostic tool is necessary. Other metabolites that are potential biomarkers of HLB disease in the asymptomatic stage are: ABA, abieta-8,11,13-trien-18-oic acid, 4-AMCH, feruloylputrescine, $p$-hydroxycinnamic Acid, isoleucine, pipecolic acid, tangeretin, and TMF.

\section{Conclusions}

Through MSI analyses, it was possible to identify metabolites that participate in the CLas infection process and plant defense, such as phytohormones, organic acids, carbohydrates, flavonoids, and amino acids that previously were reported in literature ${ }^{46,48,57,61}$. The images provide a better understanding of $\mathrm{m} / z$ distribution on the leaf's surface in the different HLB disease stages. The detection of these metabolites in asymptomatic leaves is important as a potential technique for HLB diagnosis, opening possibilities to search for new biomarkers. The metabolites abieta-8,11,13-trien-18-oic acid, suggested by GNPS, and 4-acetyl-1-methylcyclohexene showed a higher distribution in symptomatic leaves. These metabolites have been directly related with HLB disease and needs to be further investigated concerning the function in the CLas infection.

Received: 24 February 2020; Accepted: 27 July 2020

Published online: 10 August 2020

\section{References}

1. Bové, J. M. Huanglongbing: A destructive, newly-emerging, century-old disease of citrus. J. Plant Pathol. 88, 7-37 (2006).

2. Bassanezi, R. B., Montesino, L. H. \& Stuchi, E. S. Effects of huanglongbing on fruit quality of sweet orange cultivars in Brazil. Eur. J. Plant Pathol. 125, 565-572 (2009).

3. Lopes, S. A. \& Frare, G. F. Graft transmission and cultivar reaction of citrus to 'Candidatus Liberibacter americanus'. Plant Dis. 92, 21-24 (2008).

4. Hall, D. G., Richardson, M. L., Ammar, E.-D. \& Halbert, S. E. Asian citrus psyllid, Diaphorina citri, vector of citrus huanglongbing disease. Entomol. Exp. Appl. 146, 207-223 (2013).

5. Jagoueix, S., Bové, J.-M. \& Garnier, M. The phloem-limited bacterium of Greening disease of citrus is a member of the $a$ subdivision of the Proteobacteria. Int. J. Syst. Evol. Microbiol. 44, 379-386 (1994).

6. Texeira, D. C. et al. First report of a Huanglongbing-like disease of citrus in Sao Paulo state, Brazil and Association of a new Liberibacter species, “Candidatus Liberibacter americanus”, with the disease. Plant Dis. 89, 107 (2007).

7. Tannières, M., Fowler, S. V., Manaargadoo-Catin, L., Lange, C. \& Shaw, R. First report of 'Candidatus Liberibacter europaeus' in the United Kingdom. New Dis. Rep. 41, 3 (2020).

8. Molki, B. et al. Physiochemical changes mediated by “Candidatus Liberibacter asiaticus” in Asian citrus psyllids. Sci. Rep. 9, 16375 (2019).

9. Blaustein, R. A., Lorca, G. L. \& Teplitski, M. Challenges for managing Candidatus Liberibacter spp. (Huanglongbing disease pathogen): Current control measures and future directions. Phytopathology 108, 424-435 (2018).

10. Lee, J. A. et al. Asymptomatic spread of huanglongbing and implications for disease control. PNAS 112, 7605-7610 (2015).

11. Munir, S. et al. Huanglongbing control: Perhaps the end of the beginning. Microb. Ecol. 76, 192-204 (2018).

12. Junior, J. B. et al. Base científica para a erradicação de plantas sintomáticas e assintomáticas de huanglongbing (HLB, greening) visando o controle efetivo da doença. Trop. Plant Pathol. 34, 137-145 (2009).

13. Valdés, R. A. et al. A review of techniques for detecting Huanglongbing (greening) in citrus. Can. J. Microbiol. 62, 803-811 (2016).

14. Paudyal, K. P. Technological advances in Huanglongbing (HLB) or Citrus Greening disease management. J. Nep. Agric. Res. Counc. 1, 41-50 (2015).

15. Trivedi, P. et al. Quantification of viable Candidatus Liberibacter asiaticus in hosts using quantitative PCR with the aid of ethidium monoazide (EMA). Eur. J. Plant Pathol. 124, 553-563 (2009).

16. Freitas, D. S., Carlos, E. F., Gil, M. C. S. S., Vieira, L. G. E. \& Alcantara, G. B. NMR-based metabolomic analysis of Huanglongbingasymptomatic and -symptomatic citrus trees. J. Agric. Food Chem. 63, 7582-7588 (2015).

17. Pontes, J. G. M. et al. Metabolomics by NMR spectroscopy in plant disease diagnostic: Huanglongbing as a case study. ChemistrySelect. 6, 1176-1178 (2016).

18. Cardinali, M. C. B. et al. Infrared spectroscopy: A potential tool in huanglongbing and citrus variegated chlorosis diagnosis. Talanta 91, 1-6 (2012).

19. Cevallos-Cevallos, J. M., Rouseff, R. \& Reyes-de-Corcuera, J. I. Untargeted metabolite analysis of healthy and Huanglongbinginfected orange leaves by CE-DAD. Electrophoresis 30, 1240-1247 (2009).

20. Aksenov, A. A. et al. Detection of huanglongbing disease using differential mobility spectrometry. Anal. Chem. 86, 2481-2488 (2014).

21. Wang, K. et al. The early, rapid, and non-destructive detection of citrus Huanglongbing (HLB) based on microscopic confocal Raman. Food Anal. Method. 12, 2500-2508 (2019).

22. Farber, C., Mahnke, M., Sanchez, L. \& Kurouski, D. Advanced spectroscopic techniques for plant disease diagnostics. A review. Trend. Anal. Chem. 118, 43-49 (2019).

23. Emwas, A.-H.M., Salek, R. M., Griffin, J. L. \& Merzaban, J. NMR-based metabolomics in human disease diagnosis: applications, limitations, and recommendations. Metabolomics 9, 1048-1072 (2013).

24. Fernández-Maestre, R. Ion mobility spectrometry: History, characteristics and applications. Rev. UDCA Actualidad Divulgación Científica 15, 467-479 (2012). 
25. Everall, N. J. Confocal Raman microscopy: Common errors and artefacts. Analyst 135, 2512-2522 (2010).

26. Dong, Y. et al. Sample preparation for Mass Spectrometry Imaging of plant tissues: A review. Front. Plant Sci. 7, 60 (2016).

27. Bjarnholt, N., Li, B., D’Alvise, J. \& Janfelt, C. Mass spectrometry imaging of plant metabolites-Principles and possibilities. Nat. Prod. Rep. 31, 818-837 (2014).

28. Kumara, P. M., Shaanker, R. U. \& Pradeep, T. The UPLC and ESI-MS analysis of metabolites of Rauvolfia tetraphylla L. and their spatial localization using desorption electrospray ionization (DESI) mass spectrometric imaging. Phytochemistry 159, 20-29 (2019).

29. Perez, C. J., Bagga, A. K., Prova, S. S., Taemeh, M. Y. \& Ifa, D. R. Review and perspectives on the applications of mass spectrometry imaging under ambient conditions. Rapid Commun. Mass Spectrom. 33, 27-53 (2019).

30. Kumara, P. M., Srimany, A., Ravikanth, G., Shaanker, R. U. \& Pradeep, T. Ambient ionization mass spectrometry imaging of rohitukine, a chromone anti-cancer alkaloid, during seed development in Dysoxylum binectariferum Hook.f (Meliaceae). Phytochemistry 116, 104-110 (2015).

31. Freitas, J. R. L., Vendramini, P. H., Melo, J. O. F., Eberlin, M. N. \& Augusti, R. Assessing the spatial distribution of key flavonoids in Mentha $\times$ piperita leaves: An application of Desorption Electrospray Ionization Mass Spectrometry Imaging (DESI-MSI). J. Braz. Chem. Soc. 30, 1437-1446 (2019).

32. Soares, M. S. et al. Quantification and localization of hesperidin and rutin in Citrus sinensis grafted on C. limonia after Xylella fastidiosa infection by HPLC-UV and MALDI imaging mass spectrometry. Phytochemistry 115, 161-170 (2015).

33. Tata, A., Perez, C. J., Hamid, T. S., Bayfield, M. A. \& Ifa, D. R. Analysis of metabolic changes in plant pathosystems by imprint imaging DESI-MS. J. Am. Soc. Mass Spectrom. 26, 641-648 (2015).

34. Bhandari, D. R. et al. Histology-guided high-resolution AP-SMALDI mass spectrometry imaging of wheat-Fusarium graminearum interaction at the root-shoot junction. Plant Methods 14, 103 (2018).

35. Barbosa, E. A. et al. Imaging mass spectrometry of endogenous polypeptides and secondary metabolites from galls induced by root-knot nematodes in tomato roots. Mol. Plant Microbe In. 31, 1048-1059 (2018).

36. Murray, M. G. \& Thompson, W. F. Rapid isolation of higher weight DNA. Nucleic Acids Res. 8, 4321-4325 (1980).

37. Li, W., Hartung, J. S. \& Levy, L. Quantitative real-time PCR for detection and identification of Candidatus Liberibacter species associated with citrus Huanglongbing. J. Microbiol. Methods 66, 104-115 (2006).

38. Cifuentes-Arenas, J. C., Goes, A., Miranda, M. P., Beattie, G. A. C. \& Lopes, S. A. Citrus flush shoot ontogeny modulates biotic potential of Diaphorina citri. PLoS ONE 13, e0190563 (2018).

39. Fiehn, O. Metabolomics-The link between genotypes and phenotypes. Plant Mol. Biol. 48, 155-171 (2002).

40. Kumar, R., Bohra, A., Pandey, A. K., Pandey, M. K. \& Kumar, A. Metabolomics for plant improvement: Status and prospects. Front. Plant Sci. 8, 1302 (2017).

41. Lanekoff, I. et al. High-speed tandem mass spectrometric in situ imaging by nanospray desorption electrospray ionization mass spectrometry. Anal. Chem. 85, 9596-9603 (2013).

42. Wishart, D. S. et al. HMDB: The Human Metabolome Database. Nucleic Acids Res. 35, D521-D526 (2007).

43. Wang, M. et al. Sharing and community curation of mass spectrometry data with Global Natural Products Social Molecular Networking. Nat. Biotechnol. 34, 828-837 (2016).

44. Dictionary of natural products (DNP). https://dnp.chemnetbase.com (2000).Accessed 31 July 2020.

45. Asai, T., Matsukawa, T. \& Kajiyama, S. Metabolomic analysis of primary metabolites in citrus leaf during defense responses. J. Biosci. Bioeng. 123, 376-381 (2017).

46. Killiny, N. \& Nehela, Y. Metabolomic response to huanglongbing: Role of carboxylic compounds in Citrus sinensis response to 'Candidatus Liberibacter asiaticus' and its vector, Diaphorina citri. Mol. Plant Microbe Interact. 30, 666-678 (2017).

47. Hijaz, F. M. et al. An HPLC-MS Characterization of the changes in sweet orange leaf metabolite profile following infection by the bacterial pathogen Candidatus Liberibacter asiaticus. PLoS ONE 8, e79485 (2013).

48. Massenti, R., Bianco, R. L., Sandhu, A. K., Gu, L. \& Sims, C. Huanglongbing modifies quality components and flavonoid content of 'Valencia' oranges. J. Sci. Food Agric. 96, 73-78 (2016).

49. Slisz, A. M., Breksa, A. P., Mishchuk, D. O., McCollum, G. \& Slupsky, C. M. Metabolomic analysis of citrus infection by 'Candidatus Liberibacter' reveals insight into pathogenicity. J. Proteome Res. 11, 4223-4230 (2012).

50. Zaparolli, D. Biological control against citrus greening. Revista Pesquisa FAPESP 254, 60-63 (2018).

51. Dala-Paula, B. M. et al. Effect of Huanglongbing or Greening disease on orange juice quality, a review. Front. Plant Sci. 9, 1976 (2019).

52. Towers, M. W., Karancsi, T., Jones, E. A., Pringle, S. D. \& Claude, E. Optimised Desorption Electrospray Ionisation Mass Spectrometry Imaging (DESI-MSI) for the analysis of proteins/peptides directly from tissue sections on a travelling Wave Ion Mobility Q-ToF. J. Am. Soc. Mass Spectrom. 29, 2456-2466 (2018).

53. Chramow, A., Hamid, T. S., Eberlin, L. S., Girod, M. \& Ifa, D. R. Imaging of whole zebra fish (Danio rerio) by desorption electrospray ionization mass spectrometry. Rapid Commun. Mass Spectrom. 28, 2084-2088 (2014).

54. Kooijman, P. C. et al. Increased throughput and ultrahigh mass resolution in DESI FT-ICR MS imaging through new-generation external data acquisition system and advanced data processing approaches. Sci. Rep. 9, 8 (2019).

55. Hosokawa, N., Sugiura, Y. \& Setou, M. Ion image reconstruction using BioMap software. In Imaging Mass Spectrometry: Protocols for Mass Microscopy (ed. Setou, M.) 113-126 (Springer, New York, 2010).

56. Vargas, I., Sanz, I., Moya, P. \& Prima-Yúfera, E. Antimicrobial and antioxidant compounds in the nonvolatile fraction of expressed orange essential oil. J. Food Protect. 62, 929-932 (1999).

57. Nehela, Y., Hijaz, F., Elzaawely, A. A., El-Zahaby, H. M. \& Killiny, N. Citrus phytohormonal response to Candidatus Liberibacter asiaticus and its vector Diaphorina citri. Physiol. Mol. Plant P. 102, 24-35 (2018).

58. Qiao, P. et al. Characterization of aroma active compounds in fruit juice and peel oil of Jinchen sweet orange fruit (Citrus sinensis (L.) Osbeck) by GC-MS and GC-O. Molecules 13, 1333-1344 (2008).

59. Baldovini, N. \& Filippi, J.-J. Natural fragrant raw materials. In Springer Handbook of Odor (ed. Buettner, A.) 11-12 (Springer, New York, 2017).

60. Chin, E. L., Mishchuk, D. O., Breksa, A. P. \& Slupsky, C. M. Metabolite signature of Candidatus Liberibacter asiaticus infection in two citrus varieties. J. Agric. Food Chem. 62, 6585-6591 (2014).

61. Fan, J., Chen, C., Brlansky, R. H., Gmitter, F. G. Jr. \& Li, Z.-G. Changes in carbohydrate metabolism in Citrus sinensis infected with 'Candidatus Liberibacter asiaticus'. Plant Pathol. 59, 1037-1043 (2010).

62. Xu, M. et al. Transcriptional analyses of Mandarins seriously infected by 'Candidatus Liberibacter asiaticus'. PLoS ONE 10, e0133652 (2015).

63. Manthey, J. A. Differences in secondary metabolites in leaves from orange (Citrus sinensis L.) trees affected with greening disease (Huanglongbing) (HLB). Proc. Fla. State Hort. Soc. 121, 285-288 (2008).

64. Jones, S. E. et al. GC-MS analysis of secondary metabolites in leaves from orange trees infected with HLB: A 9-month course study. Proc. Fla. State Hort. Soc. 125, 75-83 (2012).

65. Uckoo, R. M., Jayaprakasha, G. K. \& Patil, B. S. Chromatographic techniques for the separation of polymethoxyflavones from citrus, vol 1093. In Emerging trends in dietary components for preventing and combating disease (eds Patil, B. S. et al.) 3-15 (ACS Division of Agricultural and Food Chemistry, Washington, 2012).

66. Sétamou, M., Alabi, O. J., Simpson, C. R. \& Jifon, J. L. Contrasting amino acid profiles among permissive and non-permissive hosts of Candidatus Liberibacter asiaticus, putative causal agent of Huanglongbing. PLoS ONE 12, e0187921 (2017). 
67. Deterre, S. et al. Chemical and sensory characterization of orange (Citrus sinensis) pulp, a by-product of orange juice processing using Gas-Chromatography-Olfactometry. J. Food Qual. 39, 826-838 (2016).

68. Hung, H.-L. \& Wang, Y. Metabolite profiling of Candidatus Liberibacter infection in Hamlin sweet oranges. J. Agric. Food Chem. 66, 3983-3991 (2018).

69. Yao, L. et al. Proteomic and metabolomic analyses provide insight into the off-flavour of fruits from citrus trees infected with 'Candidatus Liberibacter asiaticus'. Hort. Res. 6, 31 (2019).

70. Wheaton, T. A. \& Stewart, I. Feruloylputrescine: Isolation and identification from citrus leaves and fruit. Nature 206, 620-621 (1965).

71. Baldwin, E. et al. Effect of Liberibacter infection (Huanglongbing disease) of citrus on orange fruit physiology and fruit/fruit juice quality: Chemical and physical analyses. J. Agric. Food Chem. 58, 1247-1262 (2010).

72. Mercader, J., Wanecq, E., Chen, J. \& Carpéné, C. Isopropylnorsynephrine is a stronger lipolytic agent in human adipocytes than synephrine and other amines present in Citrus aurantium. J. Physiol. Biochem. 67, 443-452 (2011).

73. Fake, C. Growing citrus in the Sierra Nevada foothills. https://ucanr.edu/sites/placernevadasmallfarms/files/63813.pdf (2010). Accessed 31 July 2020.

74. Tsujita, T. \& Takaku, T. Lipolysis induced by segment wall extract from Satsuma mandarin orange (Citrus unshu Mark). J. Nutr. Sci. Vitaminol. 53, 547-551 (2007).

75. Yu, Q. et al. Proteomic and metabolomic analyses provide insight into production of volatile and non-volatile flavor components in mandarin hybrid fruit. BMC Plant Biol. 15, 76 (2015).

76. Yannai, S. Dictionary of food compounds with CD-ROM: Additives, flavors, and ingredients. 1st edn (ed. Yannai, S.) 1784 (Chapman and Hall/CRC, 2003).

77. Tao, N. G. et al. Essential oil composition and antimicrobial activity of Citrus reticulate. Chem. Nat. Compd. 45, 437-438 (2009).

78. Wang, N. \& Trivedi, P. Citrus Huanglongbing: A newly relevant disease presents unprecedented challenges. Phytopathology 103, 652-665 (2013).

79. Bari, R. \& Jones, J. D. G. Role of plant hormones in plant defence responses. Plant Mol. Biol. 69, 473-488 (2009).

80. War, A. R. et al. Mechanisms of plant defense against insect herbivores. Plant Signal. Behav. 7, 1306-1320 (2012).

81. Timpa, J. D., Burke, J. J., Quisenberry, J. E. \& Wendt, C. W. Effects of water stress on the organic acid and carbohydrate compositions of cotton plants. Plant Physiol. 82, 724-728 (1986).

82. Chen, L.-S. et al. Changes inorganic acid metabolism differ between roots and leaves of Citrus grandis in response to phosphorus and aluminum interactions. J. Plant Physiol. 166, 2023-2034 (2009).

83. Killiny, N. \& Hijaz, F. Amino acids implicated in plant defense are higher in Candidatus Liberibacter asiaticus-tolerant citrus varieties. Plant Signal. Behav. 11, e1171449 (2016).

84. Vuković, N. L., Vukić, M. D., Đelić, G. T., Kacaniova, M. M. \& Cvijović, M. The investigation of bioactive secondary metabolites of the methanol extract of Eryngium amethystinum. Kragujevac J. Sci. 40, 113-129 (2018).

85. Killiny, N. Metabolite signature of the phloem sap of fourteen citrus varieties with different degrees of tolerance to Candidatus Liberibacter asiaticus. Physiol. Mol. Plant Pathol. 97, 20-29 (2017).

86. Wallis, C. M., Wallingford, A. K. \& Chen, J. Grapevine rootstock effects on scion sap phenolic levels, resistance to Xylella fastidiosa infection, and progression of Pierce's disease. Front. Plant Sci. 4, 502 (2013).

87. López-Gresa, M. P. et al. Identification of defence metabolites in tomato plants infected by the bacterial pathogen Pseudomonas syringae. Environ. Exper. Bot. 74, 216-228 (2011).

88. Johansen, J. D., Heydorn, S. \& Menné, T. Oak moss extracts in the diagnosis of fragrance contact allergy. Contact Dermatitis 46, $157-161$ (2002).

89. Ioannidis, K., Melliou, E. \& Magiatis, P. High-throughput 1H-nuclear magnetic resonance-based screening for the identification and quantification of heartwood diterpenic acids in four Black Pine (Pinus nigra Arn.) marginal provenances in Greece. Molecules 24, 3603 (2019).

90. Lombardero, M. J., Solla, A. \& Ayres, M. P. Pine defenses against the pitch canker disease are modulated by a native insect newly associated with the invasive fungus. Forest Ecol. Manag. 437, 253-262 (2019).

91. Fukui, H., Koshimizu, K. \& Egawa, H. A new diterpene with antimicrobial activity from Chamaecyparis pisifera Endle. Agric. Biol. Chem. 42, 1419-1423 (1978).

92. Hansen, N. L. et al. The terpene synthase gene family in Tripterygium wilfordii harbors a labdane-type diterpene synthase among the monoterpene synthase TPS-b subfamily. Plant J. 89, 429-441 (2017).

93. Feio, S. S. et al. Antimicrobial activity of dehydroabietic acid derivatives (II). In Proceedings of the Phytochemical Society of Europe: Natural Products in the New Millennium: Prospects and Industrial Application Vol. 47 (eds Rauter, A. M. et al.) 241-247 (Kluwer Academic Publishers, New York, 2002)

94. Silva, K. R. et al. Antibacterial and cytotoxic activities of Pinus tropicalis and Pinus elliottii resins and of the diterpene Dehydroabietic Acid against bacteria that cause dental caries. Front. Microbiol. 10, 987 (2019).

95. Zeier, J. New insights into the regulation of plant immunity by amino acid metabolic pathways. Plant Cell Environ. 36, 2085-2103 (2013).

96. Bernsdorff, F. et al. Pipecolic acid orchestrates plant systemic acquired resistance and defense priming via salicylic acid-dependent and-independent pathways. Plant Cell 28, 102-129 (2016).

97. Li, W., Yao, Y.-N., Wu, L. \& Hu, B. Detection and seasonal variations of Huanglongbing disease in Navel orange trees using direct ionization mass spectrometry. J. Agric. Food Chem. 67, 2265-2271 (2019).

98. Smeekens, S. Sugar-induced signal transduction in plants. Annu. Rev. Plant Physiol. Plant Mol. Biol. 51, 49-81 (2000).

\section{Acknowledgements}

This study was financed in part by the Coordenação de Aperfeiçoamento de Pessoal de Nível Superior-Brasil (CAPES) -Finance Code 001, Fundação de Amparo à Pesquisa no Estado de São Paulo [Grant numbers FAPESP 2017/24462-4 and 2018/03670-0, 2019/06359-7] and L'Oréal Brazil, together with ABC and UNESCO in Brazil.

\section{Author contributions}

J.G.M.P. and L.S.F. contributed to sample preparation for LC-MS/MS and DESI-MSI analyses; P.H.V. and J.G.M.P performed DESI-MSI analyses; J.G.M.P., L.S.F. and T.P.F. contributed to data interpretation; F.H.S and E.J.P performed LC-MS/MS analyses; R.F.M. and N.A.W. contributed to plant cultivation, PCR analyses and sample collection; J.G.M.P., R.F.M., N.A.W. and T.P.F. contributed to writing the manuscript; P.H.V., E.J.P., M.N.E., N.A.W. and T.P.F. revised the manuscript.

\section{Competing interests}

The authors declare no competing interests. 


\section{Additional information}

Supplementary information is available for this paper at https://doi.org/10.1038/s41598-020-70385-4.

Correspondence and requests for materials should be addressed to T.P.F.

Reprints and permissions information is available at www.nature.com/reprints.

Publisher's note Springer Nature remains neutral with regard to jurisdictional claims in published maps and institutional affiliations.

Open Access This article is licensed under a Creative Commons Attribution 4.0 International License, which permits use, sharing, adaptation, distribution and reproduction in any medium or format, as long as you give appropriate credit to the original author(s) and the source, provide a link to the Creative Commons license, and indicate if changes were made. The images or other third party material in this article are included in the article's Creative Commons license, unless indicated otherwise in a credit line to the material. If material is not included in the article's Creative Commons license and your intended use is not permitted by statutory regulation or exceeds the permitted use, you will need to obtain permission directly from the copyright holder. To view a copy of this license, visit http://creativecommons.org/licenses/by/4.0/.

(c) The Author(s) 2020 